\title{
Bartonella henselae Endocarditis: An Usual Presentation of an Unusual Disease
}

\author{
Aasem Abu Shtaya, Shoshan Perek, Adi Kibari, Shai Cohen \\ Department of Internal Medicine 'B', Lady Davis Carmel Medical Center, Haifa, Israel
}

Received: 30/12/2019

Accepted: 05/02/2019

Published: 04/03/2019

\begin{abstract}
How to cite this article: Abu Shtaya A, Perek S, Kibari A, Cohen S. Bartonella henselae endocarditis: a usual presentation of an unusual disease. EJCRIM
\end{abstract} 2019;6: doi:10.12890/2019_001038.

Conflicts of Interests: The Authors declare that there are no competing interests.

This article is licensed under a Commons Attribution Non-Commercial 4.0 License

\section{ABSTRACT}

Bartonella species are an emerging cause of culture-negative endocarditis with more cases being diagnosed now than 25 years ago when Bartonella quintana endocarditis was first described in a patient infected with human immunodeficiency virus (HIV). Despite the disease being increasingly reported, the exact epidemiological features are not clear, with prevalence rates ranging between $2 \%$ and $10 \%$ of all cases of culture-negative endocarditis. Moreover, the mortality rate is still high, presumably because of the subacute nature and relative rareness of the disease. Bartonella endocarditis occurs more often in men, and previous valvular surgery is a major risk factor. There is insufficient data to guide definitive treatment due to the paucity of literature. A previous study demonstrated that effective antibiotic therapy for Bartonella endocarditis should include an aminoglycoside prescribed for a minimum of 2 weeks. However, the treatment strategy is a matter of expert opinion.

\section{LEARNING POINTS}

- Bartonella endocarditis is considered one of the most common causes of culture-negative endocarditis, yet tardy diagnosis of the disease is usually made, thus carrying a high mortality rate.

- Awareness of the unusual presentation of Bartonella endocarditis could potentially decrease complications and mortality rates.

- Contact with animals, and cats in particular, is a prominent risk factor for Bartonella henselae infection. Physicians should therefore routinely enquire about such exposure when endocarditis and especially culture-negative endocarditis is suspected.

- Empirical therapy should be started when culture-negative endocarditis is suspected and even when Duke criteria are not met; watchful waiting for final serological tests might be harmful, as recommended by some international authors.

- Most organisms causing culture-negative endocarditis are not covered in the initial treatment of infective endocarditis before or without pathogen identification recommended by the European Society of Cardiology.

\section{KEYWORDS}

Infective endocarditis, Bartonella, cat-scratch disease

\section{CASE DESCRIPTION}

An 84-year-old man presented to our department with general weakness, weight loss, shortness of breath, and a two-month history of chest pain. Preceding these symptoms, two months previously he had suffered a myocardial infarction with ST elevation, entailing emergent percutaneous coronary intervention and stent insertion to the left anterior descending artery.

His past medical history included hypertension, dyslipidemia, chronic renal failure, and valvular heart disease; 12 years prior to this hospitalization he underwent aortic valve replacement to a biological valve because of severe aortic stenosis. In addition, 4 months prior to 
the current admission the patient was evaluated by a hematologist for newly diagnosed anemia and thrombocytopenia with splenomegaly and was awaiting a bone marrow biopsy.

It is worth mentioning that the patient had sought medical attention 1 month prior to admission, when he had complained of general weakness, weight loss, decreased appetite and abdominal pain. A CT scan performed in the emergency department at the time was negative and he was discharged home for further investigation by a gastroenterologist.

On admission to our facility, the patient was stable and vital signs were normal without fever. Physical examination was significant for high jugular venous pressure (JVP) and peripheral pitting edema. A chest X-ray showed signs of pulmonary congestion and a new right pleural effusion without signs of pneumonia. Blood tests showed a mild known anemia, Hb 11.5 g, platelets 85,000, creatinine 2.5 (baseline around $1.5 \mathrm{mg} / \mathrm{dL}$ ), alkaline phosphatase $300 \mathrm{U} / \mathrm{L}, \mathrm{GGT} 455 \mathrm{U} / \mathrm{L}, \mathrm{CRP} 1.5 \mathrm{mg} / \mathrm{dL}$, and NT-proBNP >35,000. Having assumed the patient had acute decompensated heart failure, he was treated with an escalated dose of diuretics with minimal improvement in his general condition.

On day 2 of admission, the patient had a transthoracic echocardiogram which revealed a mobile bioprosthetic aortic valve mass with moderate aortic regurgitation and moderately reduced left ventricular function. A transesophageal echocardiogram the subsequent day revealed the same finding with measured dimensions of $1 \times 0.4 \mathrm{~cm}$ (Figs. 1 and 2). This finding was suspected to be a vegetation.

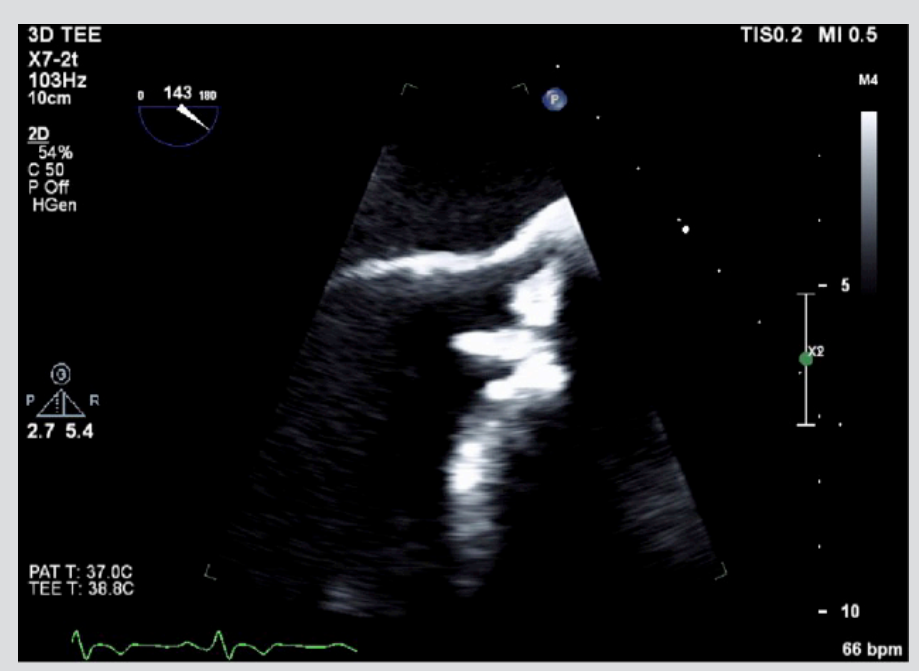

Figure 1. 2D echocardiography. A $1 \mathrm{~cm} \times 0.4 \mathrm{~cm}$ mobile bioprosthetic $66 \mathrm{bpm}$ aortic valve mass suspected to be a vegetation

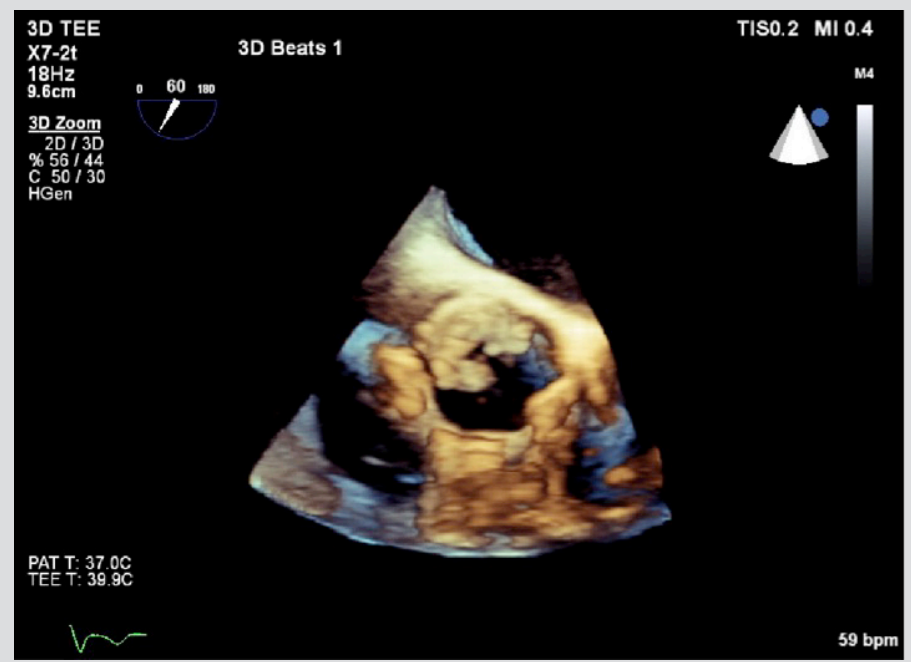

Figure 2. 3D echocardiography. The same aortic valve mass on 3D echocardiography

At this point, the patient had no fever, vital signs were stable, and blood cultures were still without growth. Serological tests were sent for Q fever, Brucella and Bartonella. Because the patient had one major criterion (a vegetation) and one minor criterion (predisposition), according to Duke criteria, antibiotic treatment was not initiated and evaluation for marantic endocarditis was carried out. Serology for antiphospholipid antibody syndrome was checked and returned negative; the rheumatological panel was negative; a total body CT was 
negative; and neoplastic markers were negative.

On day 14, serological tests for Bartonella henselae returned positive; both IgM and IgG were positive. A polymerase chain reaction (PCR) for Bartonella returned negative. Antibiotic treatment with rifampin and doxycycline was initiated; however, an aminoglycoside was not incorporated into the treatment regimen because of the patient's borderline kidney function. At this point, the patient recalled being scratched by a cat several months prior to his current admission. Subsequently, his condition acutely deteriorated, his creatinine elevated to $4 \mathrm{mg} / \mathrm{dL}$ and he became anuric, necessitating hemodialysis being performed multiple times. His liver enzymes were significantly elevated on day 16: AST was 604, ALT 204, direct bilirubin $6.5 \mathrm{mg} / \mathrm{L}$, total bilirubin $9 \mathrm{mg} / \mathrm{dL}$. Accordingly, treatment with rifampin was replaced with gentamicin. Despite treatment, there was no improvement and the patient was not considered for surgical treatment because of the high surgical risk. Hence, antibiotic coverage was continued solely without any significant improvement. On day 24 , the patient was in asystole, and considering his very poor prognosis and because of his family request and his own preferences, resuscitation was not performed. He died the same day.

\section{DISCUSSION}

Bartonella henselae infective endocarditis remains a challenging diagnosis among predisposed patients and carries a high mortality. The unusual presentation and the absence of typical infectious disease symptomatology may hinder and delay the diagnosis; a high index of suspicion is needed for early diagnosis. The disease should be suspected in patients with epidemiologic risk factors for disease whose blood cultures remain negative after 72 to 96 hours $^{[1]}$.

A detailed history and guided physical examination are necessary and could be of value to guide the diagnosis. Enquiring about any exposure to cats (Bartonella); non-pasteurized milk (Brucella); cattle, sheep, dogs and other domestic animals (Coxiella) should be routinely made as part of the history taking in the patient suspected of having endocarditis, especially when the nature of the disease is subacute. Serologic tests should be performed as early as possible in patients with negative blood cultures who have risk factors for these infections.

Cases of culture-negative infective endocarditis constitute nowadays about $10 \%$ of the overall number of cases of infective endocarditis ${ }^{[2,3]}$. With a detailed history, serologic testing, PCR and highly specialized microbiologic techniques, more than $60 \%$ of the causative organisms are identified. One of the most important drawbacks in diagnosing those patients is the relatively long period of time taken for complex serological and microbiologic techniques.

It is worth noting that the treatment guidelines for culture-negative endocarditis were incorporated into the American Heart Association (AHA) infective endocarditis guidelines 14 years ago in 2005, where most of those guidelines were derived from case reports, case series, and expert opinions. One of the reasons for the paucity of large trials on the optimal treatment of culture-negative infective endocarditis is that most of these patients require valve replacement surgery early in the course of the disease because of extensive valvular damage. In addition, the mortality rate is extremely high. Since then, no strong recommendations have been proposed.

Another reason is the poor correlation of minimal inhibitory concentration (MIC) with the in vivo efficacies of antibiotics in patients with Bartonella-related infections ${ }^{[4]}$, adding to the complexity of treatment. The MICs of most tested antibiotics are usually low, but only aminoglycosides are bactericidal[ ${ }^{[5]}$. This is observed in anoxic media and cell cultures ${ }^{[6]}$.

In 2015, the AHA endocarditis guidelines did not provide specific treatment recommendations for Bartonella endocarditis and the initial treatment recommendations proposed in 2005 are still considered the standard of care. The 2015 AHA guidelines recommend taking into consideration the country epidemiological data when treatment of culture-negative infective endocarditis is considered (Class I; Level of Evidence C); they also highly recommend consultation with an infectious disease specialist to define the most appropriate choice of therapy (Class I; Level of Evidence C).

Antibiotic therapy for endocarditis in all probability cannot be deduced from infections at other sites caused by the same microorganism. Therefore, the clinical experience in treating cat-scratch disease is probably irrelevant for Bartonella henselae endocarditis. In reviewing the literature, we concluded that selection of medical therapy for patients with culture-negative infective endocarditis is difficult. On the one hand, there is a need to provide empirical antimicrobials for all likely pathogens. On the other hand, certain therapeutic agents, including aminoglycosides, have potentially toxic effects that dictate limitation or avoidance of use if at all possible.

Moreover, some of the laboratory-based diagnostic techniques to define fastidious or unusual pathogens require considerable time for completion of testing if specimens are sent to a referral laboratory. During this period, patients are often treated empirically for the more common bacterial causes of infective endocarditis, which can result in exposure to potentially toxic therapy that could be avoided with earlier pathogen identification.

We learned through this patient in particular and other cases of culture-negative endocarditis treated in our facility that we would have incorporated an aminoglycoside into the patient's regimen despite his borderline kidney function. In retrospect, the benefit of treating the infection should have been superior to the risk of losing kidney function, taking into consideration that most reports do recommend making 


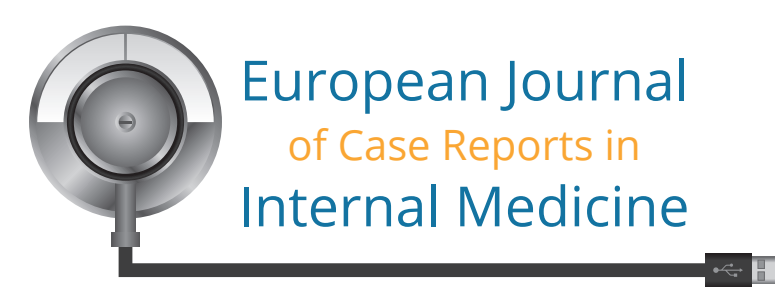

an aminoglycoside as the cornerstone of treatment.

We would have started an empirical treatment earlier, taking into consideration the 2005 AHA recommendations and the current epidemiological data in our country, even though Duke criteria were not met. However, the clinical scenario and course were suspicious. In conclusion, after the blood cultures returned negative, in retrospect, we should have looked more deeply into the causes of culturenegative endocarditis through history and physical examination along with the other diagnostic tests that were carried out (e.g. marantic endocarditis).

\section{REFERENCES}

1. Fournier PE, Lelievre H, Eykyn SJ, Mainardi JL, Marrie TJ, Raoult D, et al. 2001. Epidemiologic and clinical characteristics of Bartonella quintana and Bartonella henselae endocarditis: a study of 48 patients. Medicine (Baltimore) 2001;80:245-51. doi:10.1097/00005792-200107000-00003

2. Benslimani A, Fenollar F, Lepidi H, Raoult D. Bacterial zoonoses and infective endocarditis, Algeria. Emerg Infect Dis 2005;11: 216-24.

3. Znazen A, Rolain JM, Hammami N, Kammoun S, Hammami A, Raoult D. High prevalence of Bartonella quintana endocarditis in Sfax, Tunisia. Am J Trop Med Hyg 2005;72:50307.

4. Maurin M, Gasquet S, Ducco C, Raoult D. MICs of 28 antibiotic compounds for 14 Bartonella (formerly Rochalimaea) isolates. Antimicrob Agents Chemother 1995;39:2387-91.

5. Maurin M, Raoult D. Antimicrobial susceptibility of Rochalimaea quintana, Rochalimaea vinsonii and the newly recognized Rochalimaea henselae. J Antimcrob Chemother 1993;32:587-94.

6. Rolain JM, Maurin M, Raoult D. Bactericidal effect of antibiotics on Bartonella and Brucella spp.: clinical implications. J Antimicrob Chemother 2000;46:811-14. 